\title{
EDUCATION AND ASSESSMENT OF CLINICAL SKILLS AT TBILISI STATE MEDICAL UNIVERSITY
}

\author{
Zurab Vadachkoria, Irma Manjavidze, Marine Mamaladze, Rima Beriashvili \\ Tbilisi State Medical University, Tbilisi, Georgia
}

Introduction. Development of clinical skills and an explicit set of clinical competencies to be mastered by medical students is one of the main priorities of Tbilisi State Medical University (TSMU). TSMU is involved in the activities of MEDINE, which intends to develop guidelines and assessment criteria for achieving competency on undergraduate level in high medical schools of the countries involved in Bologna Process. The main document of MEDINE "Learning Outcomes/ Competences for Undergraduate Medical Education in Europe" [1,2] was being used as the basis for a national sectoral document in medicine and became a key standard for undergraduate medical education in Georgia.

In 2004 the Clinical Skills Center was founded at TSMU, intended to help develop and expand a clinical skills curricular process for medical students at the TSMU. Specifically, its goals are to expand clinical skills teaching and further develop a clinical skills assessment process. Achieving proficiency in the set of skills enables the medical graduate to be competent in a set of universal clinical practice abilities that will be applicable throughout a professional lifetime.

In 2007 OSCE (Objective Structured Clinical Examination) has been introduced for clinical skills evaluation of Medical students in reality based simulated environment and since 2009 - the OSCE is used for skills assessment of stomatologists. The OSCE is being conducted as an annual examination in odontology for III-V year students of the School of Stomatology [3].

On January 15-21, 2012 Tbilisi State Medical University delegation visited Oslo University (Norway) according to WHO expert, Professor Babill Stray-Pederson invitation to participate in organization and execution of OSCE. TSMU staff attended two OSCE sessions and got familiar to organizing process and all the documentation required for the exam performance. The implementation of OSCE in TSMU students' graduation exams is the next step of OSCE expansion in TSMU.

Objectives. Evaluation of OSCE materials, assessment criteria and exam environment adequacy to international standards $[4,5,6,7]$ at the faculty of Medicine and Faculty of Stomatology (Dentistry) are the main aims of the research. The main tools of the evaluation are students'/examiners' comments and satisfactory rate.
Methods. At the first stage of OSCE performance 24 medical students (12 from Georgian and 12 from English language sectors), who already had clinical skills practice about 3 months before, were randomly selected. 2 weeks before the students were given the hand-outs to get prepared for exam and after a week they had additional practice in following disciplines: Obstetrics-Gynecology, Neonatology, Pediatrics, Surgery, Cardiopulmonary auscultation, Adult Resuscitation, Adult Patient Care, Ophthalmology.

The materials for OSCE included station task (clinical case) on station door, students' sheet, special sheet for some stations for writing the answers on questions about the task and examiners evaluation sheet. Also, examiners had special evaluation toolkit to evaluate students' clinical skills, communication skills and written answers. The evaluation system was based on Norwegian model and included $65 \%$ of overall positive marks to pass the exam.

Hybrid simulators (The combination of medical simulator and simulated patient) were used in 2 OSCE stations: Station №1 - Obstetrics and Gynecology (the task included vaginal bleeding during pregnancy) and Station №6 - Lung and Heart Auscultation (the task included asthmatic symptoms and cardiac arrest).

Special questionnaire was given to students. Each student evaluated each station with 5 point system according to 6 conditions: Medical equipment and materials compliance in each station; Examiners' attitude towards students; Task content clarity; Task difficulty; Time for each task; Possibility to reveal students' skills.

OSCE examiners checklist for student performance included the following evaluation criteria: time for each station; general proficiency, taking into account procedures carried out; If station task gave possibility to evaluate students' medical knowledge; to evaluate students' communication skills and attitude to patient; the satisfactory rate form; Examiners' experience.

Visiting International WHO expert evaluated "stations" with different clinical tasks according to 3 criteria: Communication; Manipulation (Clinical Skills); Exam environment.

The OSCE was conducted as an annual examination in odontology for III-V year students of the School of

(C) Zurab Vadachkoria, Irma Manjavidze, Marine Mamaladze, Rima Beriashvili 
Stomatology to assess their clinical reasoning skills. Clinical scenarios were designed to be close to real clinical cases that students might encounter in their practice comprising (a) Phantom operative odontology, (b) Endodontic treatment of phantom tooth, (c) communication with simulated patient, and (d) analysis of the histological samples of teeth.

Results/Discussion. For students of the faculty of Medicine 12 stations were prepared at Clinical Center of TSMU . Each station had its own number and the paper with the case for students on the door. There were special arrows to show how tomove fromone station to another. Forcompleting the given task there was given 7 minutes on each station, 1 minute was given for logistics. The entrance and exit on each station was controlled by voice signal.

Overall Exam result was satisfactory for students, examiners, WHO expert and TSMU stuff. There was only one student, who failed the exam. The Success and Diagram describes the complete result of OSCE at faculty of Medicine (Diagram №1).

\section{Student Suceeded}

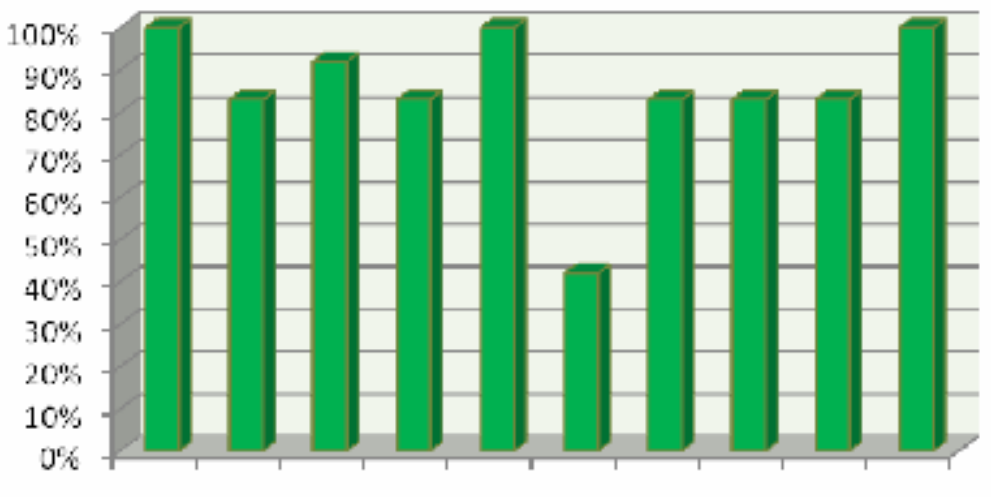

1. Obstetrics-Gynecology

2. Neonatology

3. Pediatrics

4. Surgery

5. Heart and lung auscultation

6. Resuscitation

7. Nasogastric Intubation

8. I/V catheterization

9. Urinary catheterization

10. Ophthalmology

$\begin{array}{llllllllll}1 & 2 & 3 & 4 & 5 & 6 & 7 & 8 & 9 & 10\end{array}$

\section{Diagram №1 - Students succeeded in OSCE stations}

The participant medical students, examiners and local observers were highly satisfied with the examination, and stated that the material tested was relevant, environment effective.

Statistical analyses of the Likert based items showed students had high levels of confidence in their ability to conduct physical examinations and histories, found faculty feedback helpful and informative, but were nervous throughout the examination. The qualitative analysis provided additional information regarding students' feelings about the OSCE, their levels of confidence, their stress levels and the importance of faculty feedback during the examination (Diagram №2).

\section{Average Score}

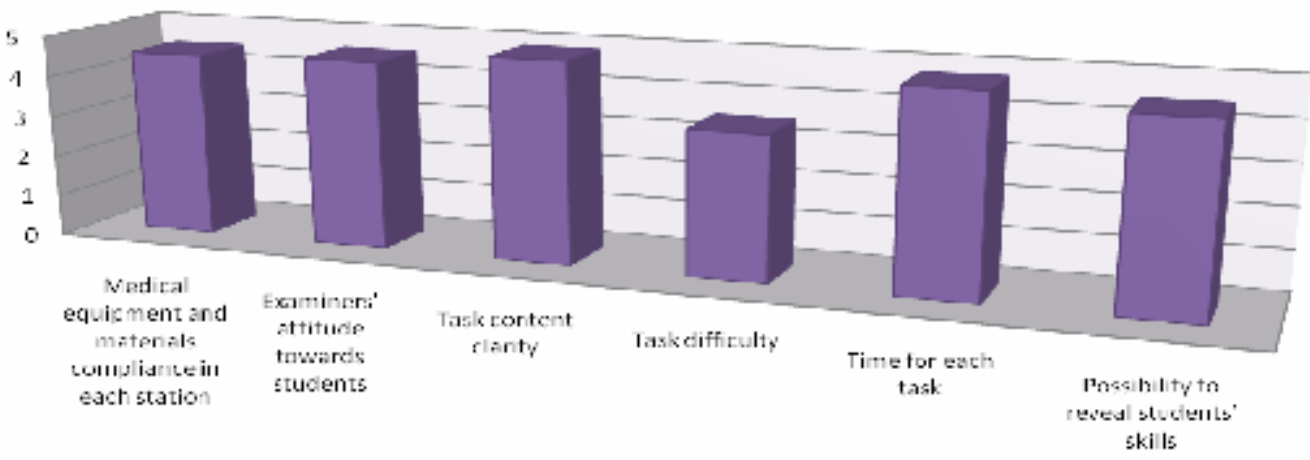

Diagram №2 - The result of student questionnaire about OSCE 
Results of examiners' questionnaire stated, that there was enough time given for all stations, except catheterization station; students' clinical skills evaluation was possible in all stations; students' medical knowledge evaluation was possible in all stations; students' communication skills evaluation was possible in four stations. All of the examiners were highly satisfied with OSCE.

The examination was assessed positively by WHO expert Professor Babil Strey-Pedersen. She noticed that the examination was well organized, objective and was conducted according to international standards. At the end, she offered the recommendations on implementation the OSCE. The main recommendation from WHO expert about modified OSCE evaluation included:

- Maximal points on each station should be the same for every station and should range from 20 to 24 ;

- The summary point of student is positive if it is $65 \%$ of maximum points (border line).

The OSCE type examinations were accepted positively by the students- stomatologists as well. Maximum objectivity of the examination reduces the doubts concerning to results of examination among the students and what is the most important it is designed to reveal the efforts, knowledge, experience and aspirations of the trainers.

Below is provided the diagrams of the exam results at the Department of Odontology (faculty of Stomatology, TSMU) on the base of the old traditional exam before (2005, 2006, 2007, 2008 years) and after $(2009,2010$, 2011, 2012 years) Objective structured clinical examination (OSCE).

\section{Before OSCE}

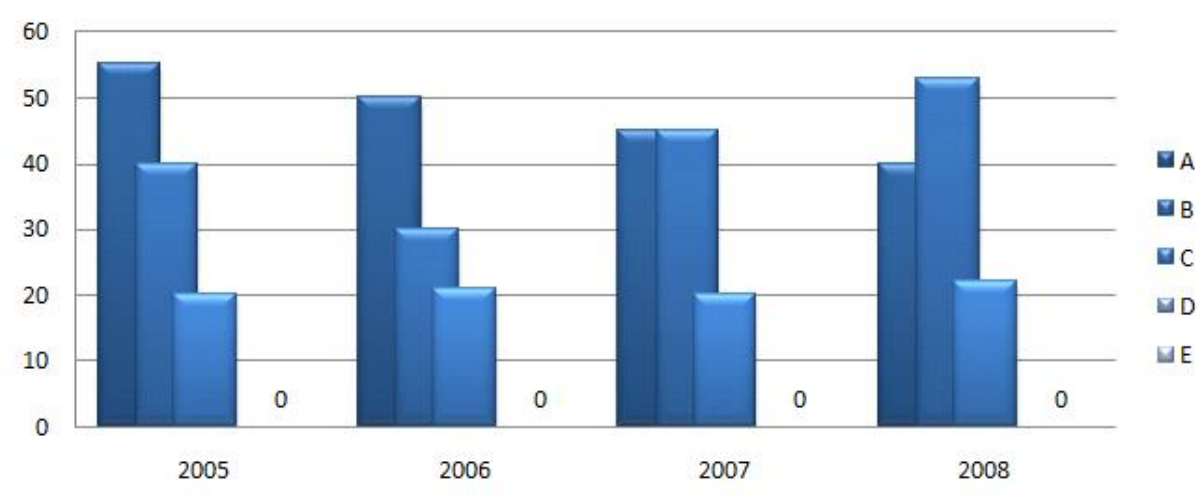

Diagram №3 - The results of students assessment at the Department of Odontology before OSCE

The results obtained after Objective Structured Clinical Examination (OSCE) at Department of Odontology revealed that every next semester the students apply much more sophisticated and refined clinical skills for patient's examination and treatment. In previous years (2005 2008) some scare and lack of knowledge the students showed at handling the clinical manipulations resulted in creation problems for their clinical activity. In addition, it should be emphasized that only a theoretical knowledge will be insufficient to prepare students for the faculty of stomatology. This is a very practical field of medicine and, therefore, the development of learning activities in this direction would be very important for the future stomatologists.

\section{After OSCE}

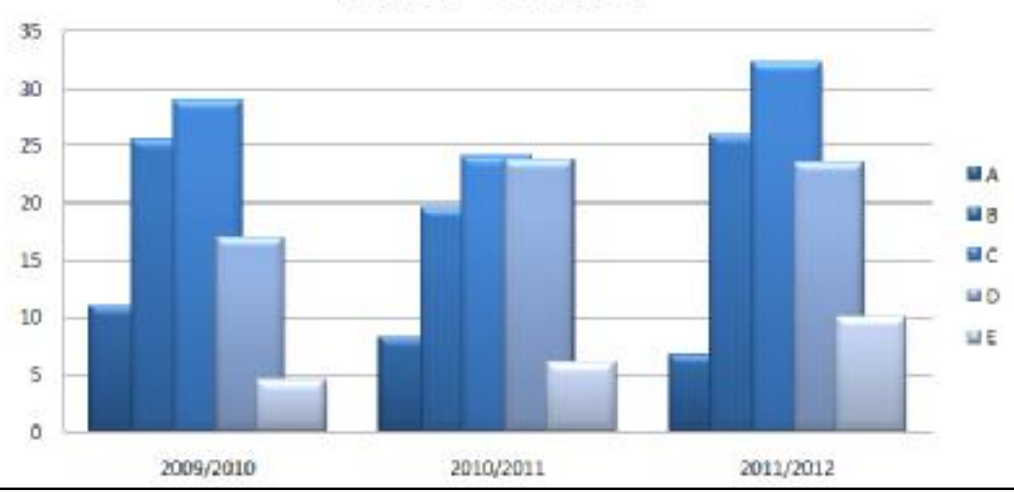

Diagram №3 - The results of students assessment at the Department of Odontology after OSCE 
Conclusion. The OSCE examination is reliable, wellestablished objective method for students' evaluation and it should be widely performed. OSCE demonstrates that student being assessed "knows how" and can "show how", allows many specific items to be assessed in a brief time. Student feedback is invaluable in influencing faculty teaching and curriculum development. The OSCE does serve to identify areas of weakness in the curriculum and teaching methods, and thus can serve as a mechanism to improve educational effectiveness.

There is no doubt that there is necessity of such type of examinations in Medicine and Stomatology, however, it is recommended to consider several advices for better performing of such examinations. Firstly, the environment

\section{References}

1. Learning Outcomes/Competences for Undergraduate Medical Education in Europe. - The Tuning Project (Medicine), 2004.

2. Harden R. M. Assessment of Medical Competence Using an Objective Structured Clinical Examination (OSCE)/ R. M. Harden, F. A. Gleeson. - ASME, Medical Education Booklet N 8. -1979.

3. Mamaladze M., Introducing OSCE for stomatology education in Georgia: experience of Tbilisi State Medical University/M. Mamaladze, N. Korsantia. -AMEE Conference Abstracts 2012. - 9CC/7.- P. 443

4. The Objective Structured Clinical Examination (OSCE) in should be comfortable that would minimize stress during the examination that is especially important in case of the simulated patients, secondly, the important part of OSCE examination is the well organized system of "feedbacks" which is another way for gaining knowledge. The short training courses for examiners are essential for gaining experience in creation of clinical scenarios and the specific assessment process of such examinations.

Application of OSCE in education and assessment appears to stimulate learning, resulting in enhancement of clinical competencies. Interviewing results revealed that more than $86 \%$ of the students consider that OSCE provides additional opportunities to demonstrate their clinical performance.

the clinical clerkship: an overview/ R. C. Tervo, E. Dimitrievich, A. L. Trujillo [et al.] // S D J Med. - 1997. - Vol. 50(5). - P. 153156.

5. Student evaluation of an OSCE in paediatrics at the University of the West Indies, Jamaica / B. P. Russell, W. Andrea, B. Michelle [et al.] // BMC Med. Educ. -2004. - Vol. 4. - P. 22.

6. Duffield K. E. A survey of medical students' views about the purpose and fairness of assessment / K. E. Duffield, J. A. Spencer // Med Edu. - 2002. - Vol. 36. - P. 879-886.

7. Surveying students' attitudes during the OSCE / R. Allen, J. Heard, M. Savidge [et al.] // Adv. Health Sci. Educ. - 1998. Vol.3.-P. 197-206. 